\title{
Can bio-plastics replace non-biodegradable plastics?
}

\begin{abstract}
Poly-3-hydroxybutyrate (PHB) is widely studied and best-characterized bioplastic within polyhydroxyalkanoates family, which can be used to produce a wide range of household and packaging products as well as medical products. Although biodegradable PHB is environmental friendly and not dependent on fossil resources, its production cost has been traditionally very expensive by bacterial fermentation techniques using recombinant $E$. coli. The recombinant diatoms and transgenic plants have also been evaluated for efficient PHB production. But, it has proved extremely difficult to increase the PHB yield that prohibits its production at the industrial scale. To address these problems, this paper has focused on the metabolic pathway manipulations in recombinant $E$. coli since they lack PHB degradation pathways unlike native producers. Another advantage of using recombinant $E$. coli is their ability to use a wide range cheap carbon sources, accumulate large amounts of polymers with higher productivity, maintain the high-cell density fermentation and recover the PHB easily. Since no single strategy has been proved to be sufficient enough to produce PHB industrially until today, the advanced and integrated approaches have to be considered for its efficient production in order to compete with non-biodegradable petrochemical plastics.
\end{abstract}

Keywords: E.coli, phb genes, transgenic plant, bacterial fermentation, nonbiodegradable, ecosystems
Volume 3 Issue 4 - 2017

\author{
Md Mohiuddin Kabir,' Chowdhury Faiz \\ Hossain, ${ }^{2}$ Kazuyuki Shimizu ${ }^{3}$ \\ 'Department of Genetic Engineering and Biotechnology, East \\ West University, Bangladesh \\ 2Department of Pharmacy, East West University, Bangladesh \\ ${ }^{3}$ Department of Bioscience and Bioinformatics, Keio University, \\ Japan
}

\begin{abstract}
Correspondence: Md Mohiuddin Kabir, PhD, Assistant Professor and Chairperson, Department of Genetic Engineering and Biotechnology, East West University, Dhaka, Bangladesh, Tel +09666775577, ext 358/359, Email mmkabir@ewubd.edu
\end{abstract}

Received: October 14, 2016 | Published: July 10, 2017
Abbreviations: PHB, poly-3-hydroxybutyrate; PHAs, polyhydroxyalkanoates; EMP, embden-meyerhof-parnas; PP, pentose phosphate; ED, entner doudoroff; SD, serine deaminase

\section{Introduction}

Polyhydroxyalkanoates (PHAs) represent a diverse group of polyesters known as bioplastics that are synthesized by many microorganisms as intracellular carbon and energy storage compounds under stress conditions. ${ }^{1}$ Since PHAs are completely biodegradable and have thermoplastic or elastomeric properties, they could offer an excellent alternative to non-biodegradable petrochemical based plastics. ${ }^{2}$ However, the production cost of biodegradable plastics is comparatively very high that limits its industrial production. As a result, the current world is heavily dependent on non-biodegradable petrochemical plastics that are very cheap. Consequently, millions of tons of non-biodegradable waste plastics are usually burned and/or dumped in landfills worldwide every year, which basically increase carbon footprint in the environment leading to global warming and natural calamities through affecting natural ecosystems. Poly-3hydroxybutyrate (PHB) is widely studied and best-characterized thermoplastic polyester within PHA family, which can be used to produce a wide range of household and packaging products such as grocery bags, soda bottles, disposable razors, flatware, etc. When they are discarded after use, the soil bacteria degrade the bioplastics naturally into water and carbon dioxide within a very short period of time. In addition, PHB and its derivatives are currently used in the medical procedures such as internal suture, vehicle for drug delivery, etc. Since bioplastics are nontoxic, biodegradable and biocompatible, they do not have to be removed after patient's recovery. PHB occurs naturally in bacteria such as Ralstonia eutropha and Bacillus megaterium that has become a model polymer for research on PHA, and for establishing and optimizing biotechnological production processes. PHB synthetic pathway genes from Ralstonia eutropha have been expressed in E. coli in order to maximize the PHB production since the bacteria E. coli do not possess PHB degradation pathways unlike native producers..$^{3-5}$ Consequently, many different fermentation strategies and downstream methods have been developed for its efficient production. ${ }^{6,7}$

The bioplastic PHB can also be produced from plants using modern molecular biology techniques. Bacterial enzymes for PHB synthesis have been introduced into the model plant Arabidopsis thaliana, but the PHB yield was extremely low. ${ }^{8}$ The main barriers of using plants are their slow growth and requirement of valuable large land area. However, biotechnological advances might improve the yield of bioplastics significantly in near future that would ultimately lead the companies to turning acres of weeds into plastic factories. To reduce the carbon footprint and the dependence on foreign oil, this new green alternative might provide an additional cash crop for farmers. The PHB encoding genes have also been expressed in diatom (Phaeodactylum tricornutum) resulting PHB accumulation into cytoplasm. The PHB yield in diatom was about $10 \%$ of the dried cell weight after seven days. ${ }^{9}$ Moreover, a cyanobacterium, Synechocystis has been reported to produce PHB when nutrients such as nitrogen become limited, which basically helps the cyanobacteria to survive under adverse conditions. However, the cyanobacteria do not naturally produce sufficient PHB. To address the limitations and opportunities for efficient PHB production, this paper has reviewed the metabolic pathway manipulations in recombinant $E$. coli since they lack PHB degradation pathways. As a result, PHB granules are not degraded in recombinant $E$. coli once synthesized. Moreover, the bacteria $E$. coli possess several advantages such as the ability to use a wide range cheap carbon sources, accumulating large amounts of polymers with higher productivity, maintaining the high-cell density fermentation and recovering PHB easily. This paper also urges to conduct more research on developing an advanced and integrated system for efficient PHB production in order to explore its feasibility at commercial scale production. 


\section{Discussion}

In native microorganisms, PHB is synthesized from acetyl-CoA by three enzymatic reactions that are catalyzed by the enzymes $\beta$-ketothiolase, acetoacetyl-CoA reductase and PHB synthase encoded by phaA, phaB and phaC genes, respectively. ${ }^{10}$ Since the whole genome and metabolic pathways of $E$. coli are known, a range of studies have been conducted to evaluate PHB production in recombinant $E$. coli. It has been found that increasing level of the precursor acetyl-CoA and the cofactor NADPH enhances the synthesis of PHB in recombinant E. coli. ${ }^{3,5}$ The principle metabolic pathways and PHB synthetic pathways in E. coli have been illustrated in Figure 1. In E. coli, most of the acetyl-CoA is synthesized from pyruvate through Embden-Meyerhof-Parnas (EMP) pathway whereas the cofactor NADPH is mainly synthesized via pentose phosphate (PP) pathway (Figure 1). Modulating the EMP pathway by over expressing the $f b a A$ gene encoding fructose bisphosphate aldolase and tpi gene encoding triosephosphate isomerase in recombinant $E$. coli had resulted higher PHB accumulation compared to the reference strains because of increased carbon flux towards the intermediary metabolite acetyl-CoA. ${ }^{7}$ It has been found that inactivation of pgi encoding enzyme phosphoglucose isomerase, which catalyzes the conversion of glucose-6-phosphate to fructose-6-phosphate, increased the pool of NADPH because of reduced carbon flux to the EMP pathway. As a consequence, most the carbon flux was channeled to PP pathway that eventually improved the PHB yield in recombinant $E$. coli since the NADPH is required in the synthesis of PHB..$^{3,5}$ The most critical enzyme trans-ketolase (encoded by $t k t A$ ) in the non-oxidative PP pathway was transformed into $E$. coli containing $p h b C A B$ operon to reinforce the metabolic flux of carbohydrate towards PHB accumulation (Figure 1). It was observed that biosynthesis of PHB was significantly increased due to carbon flux redistribution to supplement the precursor molecules acetyl-CoA. ${ }^{11}$ Moreover, the talA gene encoding trans-aldolase, another important enzyme in the non-oxidative PP pathway, was amplified in E. coli having the phb genes to modulate the metabolic flux towards the PHB biosynthetic pathway. The study showed that the PHB content in recombinant $E$. coli co-harboring the $p h b$ and talA genes was increased from $28 \%$ to $52 \%{ }^{12}$ The reason was due to the abundant supply of both NADPH and acetyl-CoA, which were basically supplemented from the activated PP pathway through the talA gene encoding enzyme transaldolase mediated modulation. Over expression of $z w f$ gene encoding enzyme glucose-6-phosphate dehydrogenase and gnd gene encoding 6-phosphogluconate dehydrogenase in oxidative PP pathway increased the pool of NADPH that ultimately enhanced the PHB synthesis in recombinant E. coli. ${ }^{13}$ Moreover, the NADP-dependent glyceraldehyde-3-phosphate dehydrogenase (encoded by gapN) from a mutant of Streptococcus, which catalyzes the conversion of glyceraldehyde-3-phosphate to glycerate-1, 3-bisphosphate, was over expressed in E. coli having $p h b$ genes. ${ }^{14}$ The result showed that the PHB accumulation was increased due to the excess supply of NADPH. Furthermore, deletion of the main acetate pathway genes (ackA and $p t a$ ) and the lactate pathway gene (ldh) in recombinant $E$. coli abolished acetate and lactate production leading to increased pool of acetyl-CoA, which eventually enhanced the PHB synthesis. ${ }^{14,15}$

In E. coli, acetyl-CoA is mostly synthesized from pyruvate catalyzed by the pyruvate dehydrogenase $(\mathrm{PDH})$ complex, which is an important intermediate metabolite in both catabolic and anabolic reactions (Figure 1). On the other hand, pyruvate is mainly synthesized through the coupled reaction of glucose uptake mediated by the phosphotransferase transport system, and through the EMP pathway and the Entner Doudoroff (ED) pathway. ${ }^{16}$ Moreover, pyruvate can also be synthesized in E. coli from L-serine via serine deaminase
(SD) pathway. ${ }^{17}$ Therefore, L-serine deaminases (encoded by the gene $s d a A$ ), catalyze the reactions to converting L-serine to pyruvate, were over expressed in recombinant E. coli. ${ }^{18}$ It was found that PHB accumulation was enhanced due to increased supply of pyruvate towards the PHB synthetic pathway via the precursor metabolite acetyl-CoA. The expression of SD and ED pathway genes together with PDH complex encoded genes (aceE, aceF and $l p d A$ ) have also been cloned in recombinant $E$. coli to evaluate the PHB production (see Figure 1 for pathways). Co-over expression of $s d a A$ gene encoding L-serine deaminase that catalyzes the conversion of L-serine to pyruvate and L-serine biosynthesis genes (SerA, $\operatorname{Ser} B$ and $\operatorname{Ser} C$ ), and $p g k$ gene encoding phosphoglycerate kinase activated the SD pathway in recombinant $E$. coli harboring $p h b$ genes from Ralstonia eutropha. ${ }^{5}$ The result showed that the PHB yield was increased by 2. 3-fold compared to the reference strain when glucose was used as the sole carbon source. Moreover, activating the ED pathway coupled with over expression of PDH complex encoded genes further enhanced the PHB production in recombinant $E$. coli due to the increased level of both pyruvate and acetyl-CoA. ${ }^{5}$

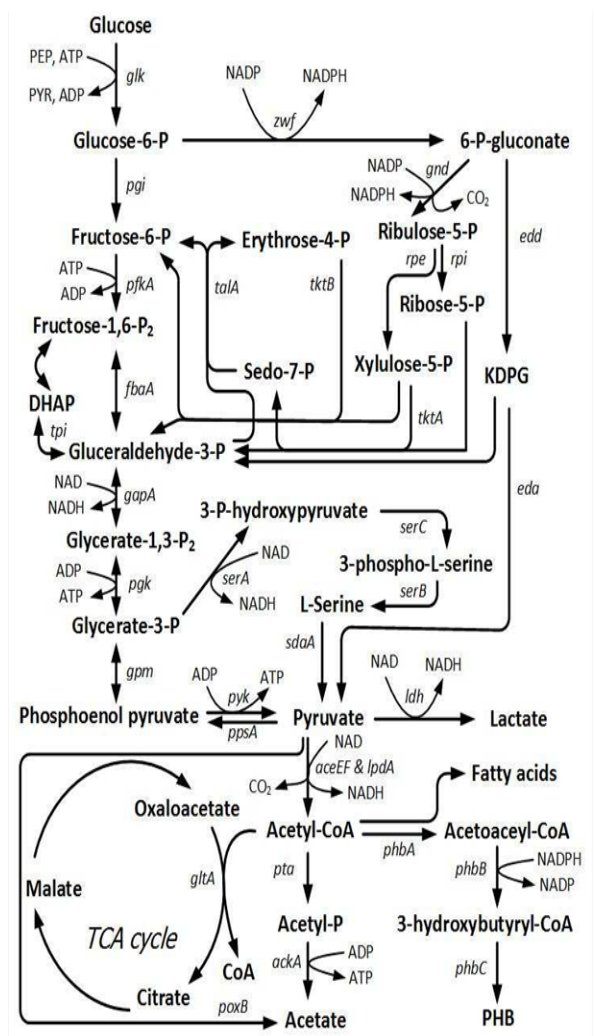

Figure I The main metabolic pathways and PHB synthetic pathways with their respective genes in recombinant $E$. coli.

KDPG, 2-keto-3-deoxy-6-phospho gluconate; DHAP, dihydroxyacetone phosphate; Sedo-7-P, sedoheptulose-7-phosphate; TCA, tricarboxylic acid; glk, glucokinase; pgi, phosphoglucose isomerase; pfkA, phosphofructokinse; fbaA, fructose bisphosphate aldolase; zwf, glucose-6-phosphate-I-dehydrogenase; gnd, 6-phosphogluconate dehydrogenase; tkt, transketolase; talA, transaldolase; edd, phosphogluconate dehydratase; eda, 2-keto-3-deoxygluconate-6phosphate Aldolase; gapA, glyceraldehyde-3-phosphate dehydrogenase; pgk, phosphoglycerate kinase; gpm, phosphoglyceromutase; sera, D-3phosphoglycerate dehydrogenase; serB, phosphoserine phosphatase; serC, 3-phosphoserine aminotransferase; sdaA, I-serine deaminase; pyk, pyruvate kinase; ppsA, phosphoenolpyruvate synthase; Idh, lactate dehydrogenase; aceE, pyruvate dehydrogenase EI; aceF, pyruvate dehydrogenase E2; IpdA, lipoamide dehydrogenase; poxB, pyruvate oxidase; $p$ ta, phosphate acetyltransferase; ackA, acetate kinase; phaA, $\beta$-ketothiolase; phaB, NADPHdependent acetoacetylCoA reductase; $\mathrm{phaC}$, $\mathrm{PHB}$ synthase; gltA, citrate synthase 


\section{Conclusion}

Millions of tons of petroleum-based plastics are produced and consumed every year worldwide generating a significant amount of waste, which have been affecting our environment adversely leading to global warming. Although PHB is biodegradable and not dependent on fossil resources, its production cost has been traditionally very expensive by bacterial fermentation techniques using recombinant $E$. coli. The recombinant diatoms and transgenic plants have also been tested for PHB production. But, it has proved extremely difficult to increase the yield of bioplastics that limits its viability for industrial production. Until today, no single strategy has been shown to be effective for the production of bioplastics in order to compete with petroleum-based plastics. Therefore, the advanced and integrated approaches must be considered for production of biodegradable plastics. One such strategy would be to explore an integrated waste water treatment plant where different types of native and/or recombinant microorganism including $E$. coli, microalgae and diatom will be harvested in sequential procedures or in mixed cultures for concomitant production of bioplastics and biofuel besides clean water (Figure 2).

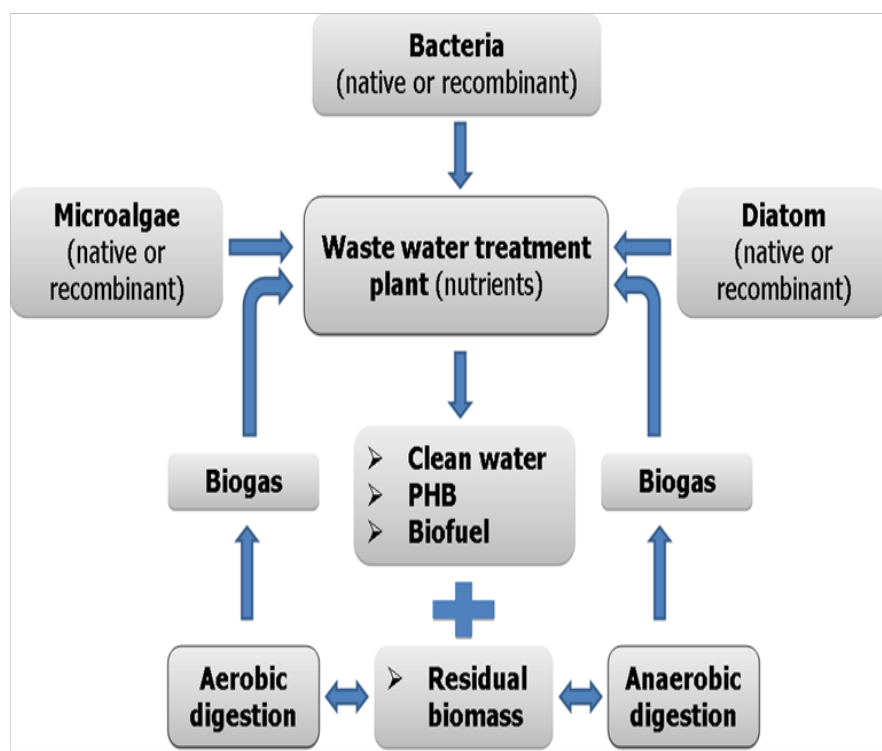

Figure $2 \mathrm{An}$ advanced and integrated waste water treatment plant to produce PHB.

\section{Acknowledgements}

None.

\section{Conflict of interest}

The author declares no conflict of interest.

\section{References}

1. Gao X, Chen JC, Wu Q, et al. Polyhydroxyalkanoates as a source of chemicals, polymers, and biofuels. Curr Opin Biotechnol. 2011;22(6):768774.

2. Choi J, Lee SY. Efficient and economical recovery of poly (3-hydroxybutyrate) from recombinant Escherichia coli by simple digestion with chemicals. Biotechnol Bioeng. 1999;62(5):546-553.
3. Kabir MM, Shimizu K. Fermentation characteristics and protein expression patterns in a recombinant Escherichia coli mutant lacking phosphoglucose isomerase for poly (3-hydroxybutyrate) production. Appl Microbiol Biotechnol. 2003;62(2-3):244-255.

4. Kabir MM, Shimizu K. Proteome analysis of a temperature-inducible recombinant Escherichia coli for poly-hydroxybutyrate production. $J$ Biosci Bioeng. 2001;92(3):277-284.

5. Zhang Y, Lin Z, Liu Q, et al. Engineering of erinedeamination pathway, Entner-Doudoroff pathway and pyruvate dehydrogenase complex to improve poly (3-hydroxybutyrate) production in Escherichia coli. Microbial Cell Factories. 2014;13:172.

6. Choi JI, Lee SY. High-level production of poly (3-hydroxybutyrate-co-3hydroxyvalerate) by fed-batch culture of recombinant Escherichia coli. Appl Environ Microbiol. 1999;65(10):4363-4368.

7. Lee SH, Kang KH, Kim EY, et al. Metabolic engineering of Escherichia coli for enhanced biosynthesis of poly (3- hydroxybutyrate) based on proteome analysis. Biotechnol Lett. 2013;35(10):1631-1637.

8. Nawrath C, Poirier Y, Somerville C. Targeting of the polyhydroxybutyrate biosynthetic pathway to the plastids of Arabidopsis thaliana results in high levels of polymer accumulation. Proc Natl Acad Sci USA. 1994;91(26):12760-12764.

9. Hempel F, Bozarth AS, Lindenkamp N, et al. Microalgae as bioreactors for bioplastic production. Microb Cell Fact. 2011;10:81.

10. Steinbüchel A, Schlegel HG. Physiology and molecular genetics of poly (betahydroxy-alkanoic acid) synthesis in Alcaligenes eutrophus. Mol Microbiol. 1991;5(3):535-542.

11. Jung YM, Lee JN, Shin HD, et al. Role of $t k t A$ gene in pentose phosphate pathway on odd-ball biosynthesis of poly-beta-hydroxybutyrate in transformant Escherichia coli harboring phbCAB operon. J Biosci Bioeng. 2004;98(3):224-227.

12. Song BG, Kim TK, Jung YM, et al. Modulation of talA gene in pentose phosphate pathway for overproduction of poly-beta-hydroxybutyrate in transformant Escherichia coli harboring phbCAB operon. $J$ Biosci Bioeng. 2006;102(3):237-240.

13. Lim SJ, Jung YM, Shin HD, et al. Amplification of the NADPH-related genes $z w f$ and gnd for the oddball biosynthesis of PHB in an E. coli transformant harboring a cloned phbCAB operon. J Biosci Bioeng. 2002;93(6):543-549.

14. Centeno-Leija S, Huerta-Beristain G, Giles-Gómez M, et al. Improving poly-3-hydroxybutyrate production in Escherichia coli by combining the increase in the NADPH pool and acetyl-CoA availability. Antonie Van Leeuwenhoek. 2014;105(4):687-696.

15. Nikel PI, Giordano AM, de Almeida A, et al. Elimination of Dlactate synthesis increases poly(3-hydroxybutyrate) and ethanol synthesis from glycerol and affects cofactor distribution in recombinant Escherichia coli. Appl Environ Microbiol. 2010;76(22):7400-7406.

16. Postma PW, Lengeler JW, Jacobson GR. Phosphoenolpyruvate: carbohydrate phosphotransferase systems of bacteria. Microbiol Rev. 1993;57(3):543-594.

17. Su HS, Lang BF, Newman EB. L-serine degradation in Escherichia coli K-12: cloning and sequencing of the sdaA gene. J Bacteriol. 1989;171(9):5095-5102.

18. Zhang X, Newman E. Deficiency in L-serine deaminase results in abnormal growth and cell division of Escherichia coli K-12. Mol Microbiol. 2008;69(4):870-881. 